\title{
Ground-based general purpose Doppler-lidar: a technology for Doppler-aerosol measurements and beyond
}

Josef Höffner, Jan Froh, Thorben Mense, Alsu Mauer, Michael Strotkamp, et al.

Josef Höffner, Jan Froh, Thorben Mense, Alsu Mauer, Michael Strotkamp, Alexander Munk, Bernd Jungbluth, Hans-Dieter Hoffmann, "Ground-based general purpose Doppler-lidar: a technology for Doppler-aerosol measurements and beyond," Proc. SPIE 11852, International Conference on Space Optics - ICSO 2020, 1185229 (11 June 2021); doi: $10.1117 / 12.2599364$

SPIE Event: International Conference on Space Optics - ICSO 2021, 2021, Online Only 


\section{International Conference on Space Optics-ICSO 2020}

Virtual Conference

30 March-2 April 2021

Edited by Bruno Cugny, Zoran Sodnik, and Nikos Karafolas
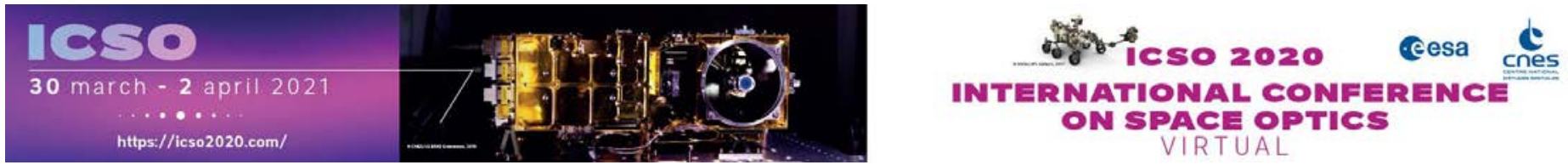

\section{Ground-based general purpose Doppler-lidar: a technology for Doppler-aerosol measurements and beyond}

\section{Cesa isoporecestings denes}




\title{
Ground-based general-purpose Doppler lidar: A technology for Doppler aerosol measurements and beyond
}

\author{
Josef Höffner*a, Jan Froh ${ }^{\mathrm{a}}$, Thorben Mense ${ }^{\mathrm{a}}$, Alsu Mauer ${ }^{\mathrm{a}}$, Michael Strotkamp ${ }^{\mathrm{b}}$, Alexander Munk ${ }^{\mathrm{b}}$, \\ Bernd Jungbluth ${ }^{\mathrm{b}}$ and Hans-Dieter Hoffmann ${ }^{\mathrm{b}}$ \\ ${ }^{a}$ Leibniz Institute of Atmospheric Physics, Schlossstraße 6, 18225 Kühlungsborn, Germany; \\ ${ }^{\mathrm{b}}$ Fraunhofer Institute for Laser Technology, Steinbachstr. 15, 52074 Aachen, Germany
}

\begin{abstract}
We present the mission proposal for a spaceborne multi-frequency lidar that is able to perform simultaneous Doppler Rayleigh, Mie and resonance measurements to gain temperature and wind data from ground to thermosphere. The suitability of the technology is demonstrated with a novel ground-based lidar with a diode-pumped alexandrite laser that acts as a unit for a lidar network able to cover the atmosphere up to $100 \mathrm{~km}$ over a large area at day- and nighttime in polar regions.

The performance of a spaceborne lidar at the iron resonance line at $386 \mathrm{~nm}$ is derived from simulations with parameters similar to Aeolus. The multi-frequency lidar achieves the same resolution and uncertainties in wind measurements as Aeolus but additionally provides the wind and temperature up to $120 \mathrm{~km}$. The reduction of the solar background by three to five orders of magnitude by means of the usage of narrow bandwidth filters and laser allows for significantly lower laser pulse energies $(\sim 10 \mathrm{~mJ})$ and higher repetition rates $(\sim 500 \mathrm{~Hz})$. Thereby the laser as a key-component and with the highest risk of failure is reduced in complexity, as no amplification stages are necessary to yield the high pulse energy.

The roadmap to space is discussed in light of the heritage from Aeolus and with the diode-pumped alexandrite laser that is in line with the FULAS (Future Laser System) platform that is also the basis for the emitter of Merlin. The necessary development steps are identified and the current activities to address them are described.
\end{abstract}

Keywords: spaceborne lidar, wind lidar, aerosols, Doppler lidar, resonance lidar, scanning lidar, diode-pumped alexandrite laser, AMD-Aeolus

\section{INTRODUCTION}

Doppler aerosol measurements, as demonstrated from space by ADM-Aeolus [1], contribute significantly to our understanding of the earth's global wind system in the troposphere and lower stratosphere. Detailed information from this previously inaccessible altitude will significantly improve midterm weather forecasts. A consequent next step are measurements in the stratosphere and mesosphere, yielding basis for improved long-term weather forecasts and improved global atmospheric models [2][3]. The ground-based Doppler iron lidar of the Institute of Atmospheric Physics (IAP) [4][5] uses a different technology for aerosol observations up to $\sim 85 \mathrm{~km}$ altitude (ice particles, NLC). NLC observations require the capability to observe lower signal strength compared to backscatter from stratospheric aerosols, observing at daytime with the challenge of high solar background. Additionally, simultaneous Doppler resonance measurements of temperature and wind are obtained in this altitude, by tuning the laser from pulse to pulse over an atomic line.

In recent years IAP and the Fraunhofer Institute of Laser Technology (ILT) have developed a technology allowing a general-purpose compact Doppler lidar within a volume of $\sim 1 \mathrm{~m}^{3}$ [6][7][8][9]. The heart of the system is a novel diodepumped alexandrite ring laser with a linewidth of $<5 \mathrm{MHz}$, tunable over a wide range $(700-800 \mathrm{~nm})$. Designed as a multi-frequency lidar it is programmable to different wavelengths from pulse to pulse. Doppler aerosol observations of wind and aerosols in the stratosphere are one of the applications of such a general-purpose lidar.

*hoeffner@iap-kborn.de; phone +49 38293 68-130; iap-kborn.de 
We demonstrate that a multi-frequency lidar with an ultra-narrow bandwidth spectral filter in the order of the linewidth of the laser has several advantages for Doppler aerosol wind measurement. It allows a highly effective rejection of the Rayleigh background and an improved sensitivity for Doppler shift measurements. Moreover, the combination of multifrequencies and period filter allows Doppler shift measurements independent of its relative amplitude. When implemented in a spaceborne lidar such a combination has the capability to obtain Doppler shift measurements in both flight direction and perpendicular, even though the shift differs largely due to the high velocity of the satellite, which is a major advantage of this technique. Beside a higher sensitivity for aerosol observations, Doppler wind observations require less photons due to the multi-frequency capability and matched spectrum of laser and spectral filter.

Spaceborne systems based on this novel technology show the potential to be used in large numbers on conventional weather satellites or as small satellites by significantly reducing size, mass and energy requirements. Compared to today's technology - which, due to its complexity and size, is foreseeable to be used only within the framework of individual, specialized science missions such as Aeolus, and thus achieve a low level of simultaneous coverage - this will allow a much more closely meshed real-time recording of processes in the atmosphere in the future.

As a first implementation of the technology, a small ground-based network for the polar region is under construction with the goal to probe the middle atmosphere from $10-100 \mathrm{~km}$.

In this paper we first describe the measurement principle of scanning and multi-frequency lidars in comparison to lidars with an emitter at a single fixed and stabilized wavelength. We also give insight in the techniques and components required for such a lidar and the benefit of a reduced effort for the spectral emitter stabilization.

Afterward, we present the current state-of-the-art for ground-based scanning frequency lidars with a compact and autonomous system that is capable of simultaneous Doppler Rayleigh, Mie and resonance measurements. The emitter bases on an efficient and robust diode-pumped alexandrite laser with a narrow linewidth. The lidars are designed to be part of a lidar network with a coverage of $\sim 100,000 \mathrm{~km}^{2}$. As exemplary results from the first field campaign wind measurements via Doppler aerosols are presented.

For a proposed spaceborne multi-frequency lidar that is able to measure the whole atmosphere from the ground to the lower thermosphere, the optimal lidar wavelength is derived. The achievable uncertainties are compared, and the suppression of the solar background is also taken into account and compared with Aeolus as a benchmark.

In the final section we describe the actual roadmap to space with the current TRL and the necessary development of certain aspects, e.g. energy-scaling and frequency conversion, of the laser as the most critical part of the instrument. The current activities with first results and designs are shown in more detail.

\section{MEASUREMENT PRINCIPLE OF SCANNING AND MULTI-FREQUENCY LIDARS}

Based on a flashlamp-pumped alexandrite ring laser a containerized mobile Doppler resonance lidar for the mesosphere has achieved Doppler potassium temperature measurements on a ship in 1996 [10]. Even though the moving and strongly vibrating platform has disturbed the laser and it was impossible to control the frequency of the laser better than several hundred MHz from pulse to pulse, we achieved Doppler temperature measurements. The mobile lidar was the first Doppler potassium lidar worldwide, operating at the potassium resonance line at $770 \mathrm{~nm}$ [11]. Earlier atmospheric test measurements at the stronger potassium line at $766 \mathrm{~nm}$ were not successful because of strong absorption in the troposphere.

At atmospheric temperatures, the typical linewidth of the atomic line is $1,000 \mathrm{MHz}$. A change of $1 \mathrm{~K}$ in the temperature broadens the line by $700 \mathrm{kHz}$. In general, it is believed that only a laser with high frequency stability can perform such Doppler measurements. Since Doppler temperature and wind measurements require at least 3 frequencies and the laser must change the frequencies from pulse to pulse it seems to be hopeless to obtain any reliable measurements with a laser not able to control the frequency at least with a few MHz accuracy. Changing the frequency from pulse to pulse is required due to strong variations of the metal density on very short time scales resulting in similar requirements of a fast-moving space borne lidar.

To obtain reliable temperature data a large etalon is used to measure the frequency of each single laser pulse in real time by imaging the interference pattern on a camera. With known frequency each measurement is a random probing of the atomic line. Instead of operating the laser at given frequencies, the measurements sample the atomic line at random frequencies. Since we know the frequency of any pulse with high precision afterwards, it is possible to repeat the measurement in the analysis if we obtain the frequency and atmospheric profile of each single laser pulse simultaneously. The achievable accuracy is finally given by the precision of the frequency measurement. The frequency jitter from pulse to pulse of the laser, which is in general characterized as rms value by operating the laser on a single frequency, has no meaning for such a lidar. 
The key is therefore the frequency measurement per laser pulse and the mathematic approach to obtain the spectrum out of the measured series of intensity versus frequency. Since the frequency is measured with $\sim 10 \mathrm{kHz}$ resolution from pulse to pulse we achieve a much higher accuracy at the end since we are not limited by the stability of the laser anymore. We note that the stability of such lasers depends on the environment and may change at any time as result of external disturbances.

Figure 1 shows on the right side the measured frequencies of 10,000 pulses of the flashlamp-pumped alexandrite ring laser operating on a single frequency. Doppler temperature measurements do not require any knowledge of the absolute frequencies since the temperature is only given by the spectral width of the atomic line, which is a relative measurement. With the additional reference of a diode laser that is stabilized on saturation spectroscopy of rubidium, shown on the left side, very high stability can be achieved over long periods. Since the average vertical velocity of the atmosphere is in the order of a few mm/s or cm/s, any reference is sufficient for Doppler wind measurements since we can calculate absolute frequencies afterwards in the analysis if the lidar is looking at least once vertical. Therefore, the frequency reference can be at any wavelength and the atmosphere serves as absolute frequency standard.

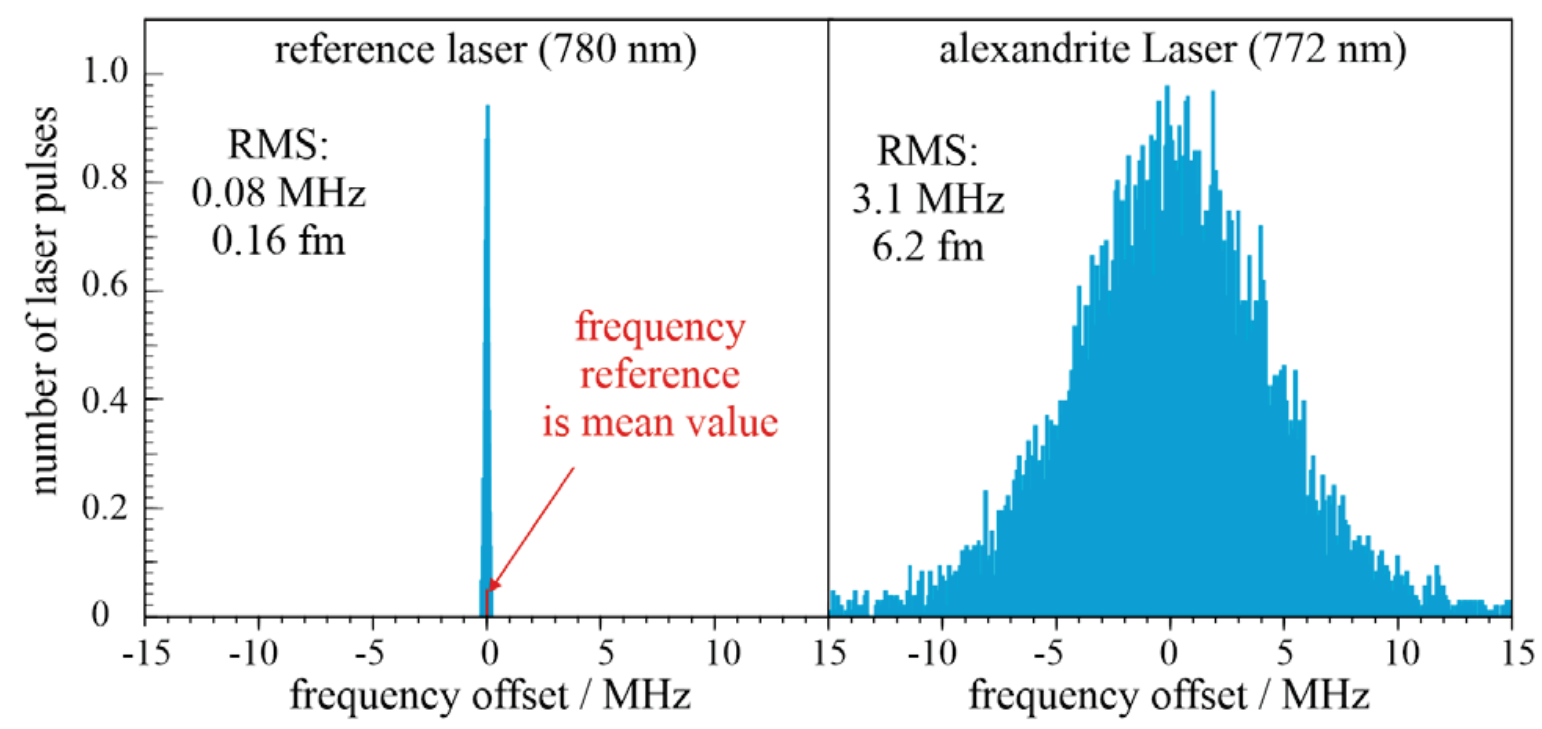

Figure 1: Relative frequency measurements of two lasers. Left side: diode laser stabilized on saturation spectroscopy of Rubidium. Right side: flashlamp-pumped alexandrite ring laser operating on a single frequency at $772 \mathrm{~nm}$.

The reference we use nowadays for Doppler measurements in the UV is saturation spectroscopy of Rubidium at $780 \mathrm{~nm}$ as shown in Figure 1. For an atomic line like iron $(386 \mathrm{~nm}$ or $372 \mathrm{~nm})$, a similar technology is not available but also not needed. Because only high frequency stability is required, we do not need any reference on the measured atomic line. We converted the former potassium lidar into the worldwide first iron Doppler lidar in 2002 based on this technology [4].

From a mathematical point of view, the measurements in 1995 are a random scanning lidar. Such a lidar requires only a stability of the laser in the order of the Doppler width of the atomic line. Figure 2 shows such a measurement. Over long periods, the lidar obtains measurements on as many frequencies as can be distinguished by the frequency measurement. Since the atomic line has a FWHM of approx. $1,000 \mathrm{MHz}$ and the frequency measurements is in the order of $10 \mathrm{kHz}$ the lidar has roughly 100,000 different frequencies. We sample only a subset of possible frequencies during a single measurement because of the limited number of laser pulses.

For spectral measurements of very narrow spectra, such as Doppler Mie, a better frequency control of the laser is required. With a ramp and fire technology, stabilizing the cavity of the laser within a single laser pulse, it became possible to operate the laser with much higher frequency stability in 1997 as already shown in Figure 1. In contrast to the measurements before it is now possible to operate the laser on discrete frequencies with gaps in between. However, a single frequency is still a mathematical idealization and of no relevance. In reality, the laser is operating at discrete frequencies with a frequency jitter shifting the frequency slightly from pulse to pulse according to Figure 1 right side. A single 
frequency is nothing else than a mathematical idealization which can only be achieved for a real system within certain limits.

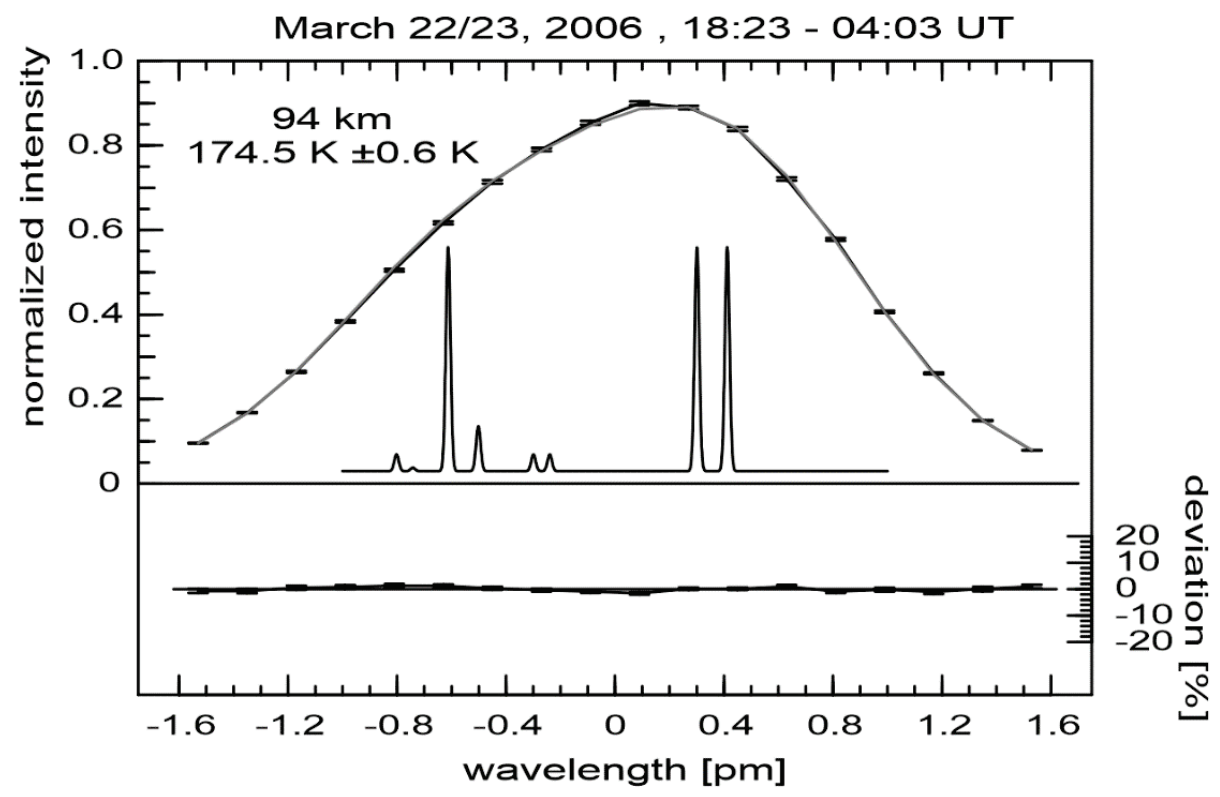

Figure 2: Doppler resonance temperature measurements at $770 \mathrm{~nm}$ (potassium) with a lidar sampling the atomic line continuously (scanning lidar). In a first step, we bin nearby frequencies in channels (points with error bars). We calculate the theoretical line shape (fitted line) for each measurement by repeating in the analysis each single measurement in the same way. The shown theoretical line shape considers the sampling of this particular measurement. The atomic line shape of potassium is given by two major isotopes each showing a hyperfine splitting. For comparison, we show the spectrum with the natural linewidth of the transitions involved.

The main difference is the first step in the mathematical analysis, which changes from a continuous spectrum to a spectrum, measured at unequal sampled frequencies. Each frequency represents a histogram of nearby laser pulses with gaps in between. We call this type of lidar a multi-frequency lidar. By controlling the laser frequency with high accuracy, a lidar can measure any number of discrete frequencies and we can therefore optimize the lidar according to the different requirements of a Doppler Rayleigh, Doppler Mie and Doppler resonance lidar. Still, we benefit from the direct measurement of the frequency of each single laser pulse as mentioned before. Within this paper, we focus for simplification on equal spacing of frequencies even though more advanced schemes allow for optimizing the lidar for different tasks. We will show that such a lidar can obtain Doppler Rayleigh, Mie and resonance measurements from space with parameters similar to the ADM-Aeolus mission if based on a diode-pumped alexandrite ring laser.

\section{NOVEL GROUND-BASED SCANNING LIDAR}

\subsection{Instrument}

The IAP replaces currently the former mobile Doppler lidar by a network of Doppler lidars based on a diode-pumped Alexandrite laser [8]. The main goal of this network called VAHCOLI (Vertical And Horizontal COverage by LIdar) is 4D-sampling of the atmosphere by a network of identical mobile state-of-the-art Doppler lidars. Each lidar-unit is currently extended to five field of views and is designed for simultaneous Doppler Rayleigh, Doppler Mie and Doppler resonance measurements, by applying more complex schemes as discussed before to obtain all spectra simultaneously. The units are designed for measurement at full daylight and contain two interferometers in the receiver and two detectors to cover the altitude range from the troposphere to the thermosphere. Even though of similar design compared to Aeolus, major differences exist. 
The goal is the operation of several such lidar systems as a Doppler lidar network which would be able to measure dynamic processes like the propagation of gravity waves through several atmospheric layers on a large scale by means of overlapping measurement ranges. The main objectives of the development of the lidar instrument were its compactness, autonomy and low price. Therefore, most components are off-the-shelf instead of customized developments, leading to drastically reduced costs with only slightly reduced performance and efficiency.

To contribute to the weight and cost target, a large fraction of the structural parts of the instrument is 3D-printed with a carefully chosen combination of standard, engineering and high-performance thermoplastics. The lidar system is well insulated and shock absorbing to provide a vibration-free environment with high temperature stability. The lidar system is equipped with systems for autonomous measurement operation such as an automated roof hatch for rough environment, or an uninterruptible power supply (UPS), which is important for safe laser operation and lockdown. The lidar can be controlled remotely via internet while attached to a standard $230 \mathrm{~V}$ wall-plug with an overall power-consumption of $\sim 500 \mathrm{~W}(\sim 1 / 3$ for an off-the-shelf PC).

For the well-established mobile narrow linewidth lidar, that was utilized for over 20 years by the IAP [4][5], the flashlamp-pumped alexandrite laser (including the cooling peripherals) was the upmost driver in power $(\sim 25 \mathrm{~kW})$ and subsequent space $\left(6 \times 2.5 \times 2.5 \mathrm{~m}^{3}\right)$ consumption and maintenance effort (flashlamps including driver). Based on this technology, a reduction in size and increase of efficiency for a lidar network or a future spaceborne lidar is not possible. Therefore, a diode-pumped alexandrite laser was developed over the last 10 years in cooperation of IAP and ILT, enabling a reduction in size of the lidar system of a factor of 40 and increase of electro-optical efficiency of a factor of 200 [5].

The available space around the telescope tube for the $50 \mathrm{~cm}$-diameter main mirror is partitioned in three vertical sections, with the middle and upper sections temperature-controlled by a constant airflow so that no misalignment occurs when the cube is operated at varying ambient temperatures. Additionally, the air in the upper section is filtered to reduce containment of the highly loaded optics by dust particles. The upper section contains the resonator unit of the laser emitter as well as the beam path for beam shaping and monitoring of the output beam. The middle section contains the pump unit of the laser emitter and the optics and electronics necessary for analyzing the backscattered signal. The lower section contains the drivers for the pump diodes and the seeder and the PC for instrumentation control and signal analysis. A cooling system for polar regions with temperatures below $20^{\circ} \mathrm{C}$ is included. The lidar operates currently at $33 \mu \mathrm{rad}$ field of view with a laser divergence of $15 \mu \mathrm{rad}$. Since the optical design was optimized for a 3D-printed and therefore instable system a movement of the telescope due to thermal heating by the sun is within limits unimportant. Test measurement show that no steering of the laser is required for many hours even though operating with a small field of view.

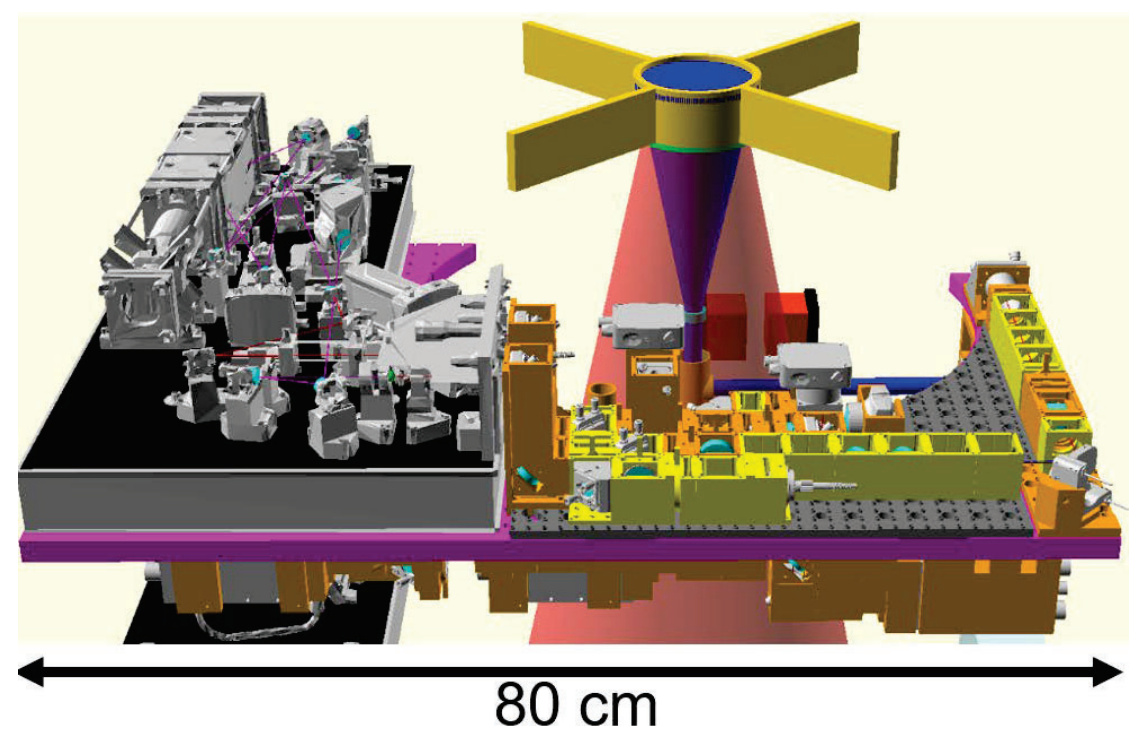

Figure 3: Diode-pumped alexandrite ring laser (left side) with 3D-printed optical system for receiver, beam steering, frequency reference and other subsystems. Yellow parts are alignment tools and are not required otherwise. The receiver hanging below is approx. $15 \times 15 \times 80 \mathrm{~cm}^{3}$ and is designed for simultaneous Doppler Rayleigh, Mie and resonance measurements. 
Details on design and manufacturing of the lidar system as well as on the development of a Doppler lidar network consisting of several lidar systems as presented here can be found in [8]. Figure 3 shows the optical system, which we built especially for the remaining space left by the telescope. Laser, Doppler free spectroscopy, beam steering and other optics for pulsed laser and seeder are on top. Below is the receiver with the double interferometer. Besides the laser, we build the whole optical system by a patent pending 3D-printed optical system with only a few commercial kinematic mounts for alignment purposes. The receiver contains two interferometers with approx. 1,000 MHz and 7.5 MHz spectral width and high finesse of 80 and 130, respectively. Next we focus on the laser technology.

\subsection{Diode-pumped alexandrite ring laser for VAHCOLI}

The laser emitter consists of a fiber-coupled pump unit and a resonator unit, detailly described in [9]. The units are built on two separate customized honeycomb breadboards. By using fiber-coupling for the pump light the both units are spatially decoupled. Thereby, they can be placed independently within the limited space of the lidar system (see Figure 4) and allow for easy replacement in case of failure of one of the modules. Additionally, the homogenization of the pump profile yields higher optical-optical energy efficiency of the resonator that compensates the losses of the fiber-coupling. A detailed analysis of the benefits of fiber-coupling for the alexandrite laser is shown in [12].

The pump unit contains two commercially available water-cooled laser diode modules emitting at $636 \mathrm{~nm}$ and tailored beam shaping optics for fiber-coupling. Due to the asymmetric beam quality of the diode modules in fast- and slow-axis (for a detailed analysis of the diode modules see [13]) beam shaping and symmetrization of the beam quality is necessary for a sufficient fiber-coupling efficiency. The optical design of the pump unit is similar to the one presented by our group in [12]. To increase the robustness against misalignment and reduce contamination by dust particles, the beam line is enclosed, and the optics are placed in specially designed, integrated mechanical mounts.

The resonator is a ring with one alexandrite crystal that is end-pumped by the fiber-coupled pump light. As the absorption of the pump light is strongly polarization-dependent and the pump light from the fiber is unpolarized, pump light back-folding is used. By means of a Faraday rotator, a unidirectional emission is guaranteed for the ring resonator. Q-switching of the resonator is achieved with a Pockels cell, waveplates for polarization adjustment and TFPs. The resonator is seeded with a narrow bandwidth $(\sim 100 \mathrm{kHz}) \mathrm{cw}$ diode laser and the cavity length is stabilized with a mirror mounted on a piezo module.

The parameters of the laser are summarized in Table 1 and a photo of the resonator in the lidar system with a transparent cover for the photo is shown on the right side of Figure 4.

Table 1. Required and measured parameters of the Alexandrite laser.

\begin{tabular}{|l|c|c|}
\hline Parameters & Ground-based requirements & Achieved \\
\hline Pulse energy & $>1 \mathrm{~mJ}$ & $1.75 \mathrm{~mJ}$ \\
\hline Repetition rate & $>150 \mathrm{~Hz}$ & $500 \mathrm{~Hz}$ \\
\hline Electro-optical efficiency & $>1 \%$ & $2 \%$ \\
\hline Pulse duration & $400-1,000 \mathrm{~ns}$ & $780 \mathrm{~ns}$ \\
\hline Wavelength & $769.898 \mathrm{~nm}$ (potassium resonance line in air) & $769.898 \mathrm{~nm}$ \\
\hline Linewidth & $\sim 99 \%<30 \mathrm{MHz}$, single-mode (lorentz) & $3.3 \mathrm{MHz}$ \\
\hline Frequency shift & $<50 \mathrm{MHz}$ & $<1 \mathrm{MHz}$ \\
\hline Frequency jitter & $<10 \mathrm{MHz}$ & $<1 \mathrm{MHz}$ \\
\hline Beam quality & $\mathrm{M}^{2}<1.5$ & $\mathrm{M}^{2}<1.1$ \\
\hline Pointing stability & $<10 \mu \mathrm{rad}$ & $\sim 3 \mu \mathrm{rad}$ \\
\hline
\end{tabular}



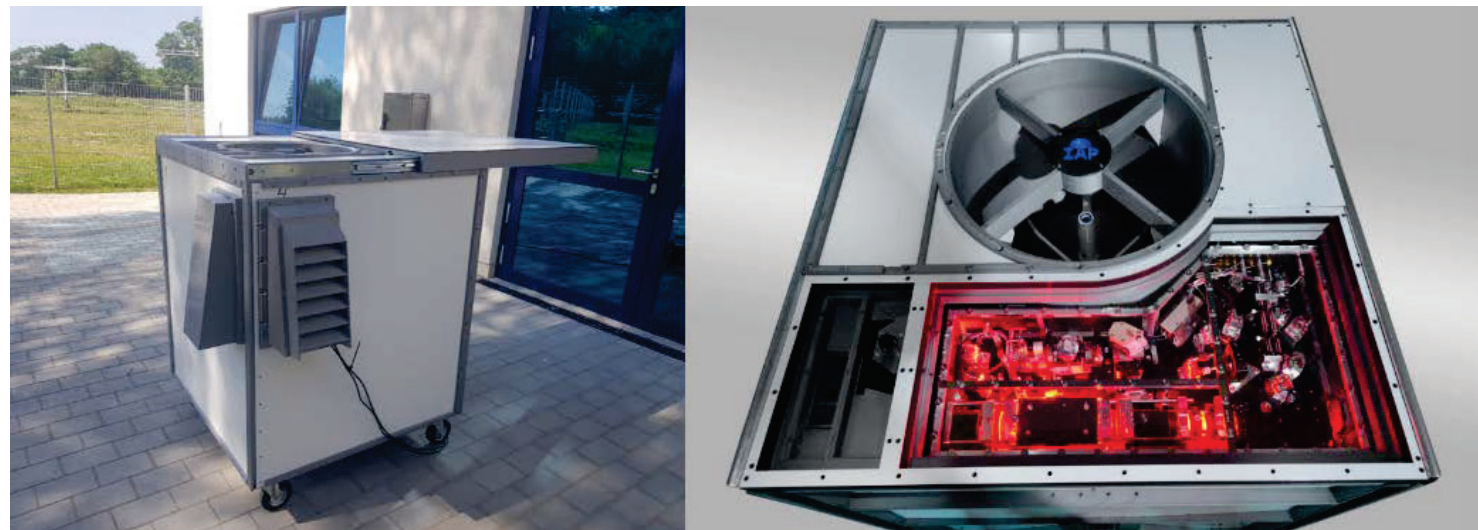

Figure 4: Photo of the lidar system measuring during the field campaigns in Kühlungsborn (left) and the operating resonator unit of the alexandrite laser under the removed cover (right).

\subsection{Results of the first field campaign}

As a first field campaign a prototype of a VAHCOLI unit was operated over several months at the IAP (shown on the left side of Figure 4). Figure 5 shows a first Doppler aerosol wind measurement in the troposphere and stratosphere. At the same time the potassium layer was observed even though the optics of receiver and telescope have been preliminary and only a low efficiency is achieved. More details about this measurement is given in [8][8][9]. The Doppler wind is finally measured by tilting the lidar approx. only $2^{\circ}$. Nevertheless, we have achieved a good agreement with ECMWF. By tilting only $2^{\circ}$ we observe roughly $4 \%$ of the Doppler effect of the horizontal wind and must therefore be able to measure small Doppler shifts with high precision. For the measurement shown here a 20 min integration time is required which is caused by the low efficiency of the receiver and the small Doppler shifts. The typical uncertainty is in the order of $20 \mathrm{~cm} / \mathrm{s}$ with this configuration at $200 \mathrm{~m}$ altitude resolution.
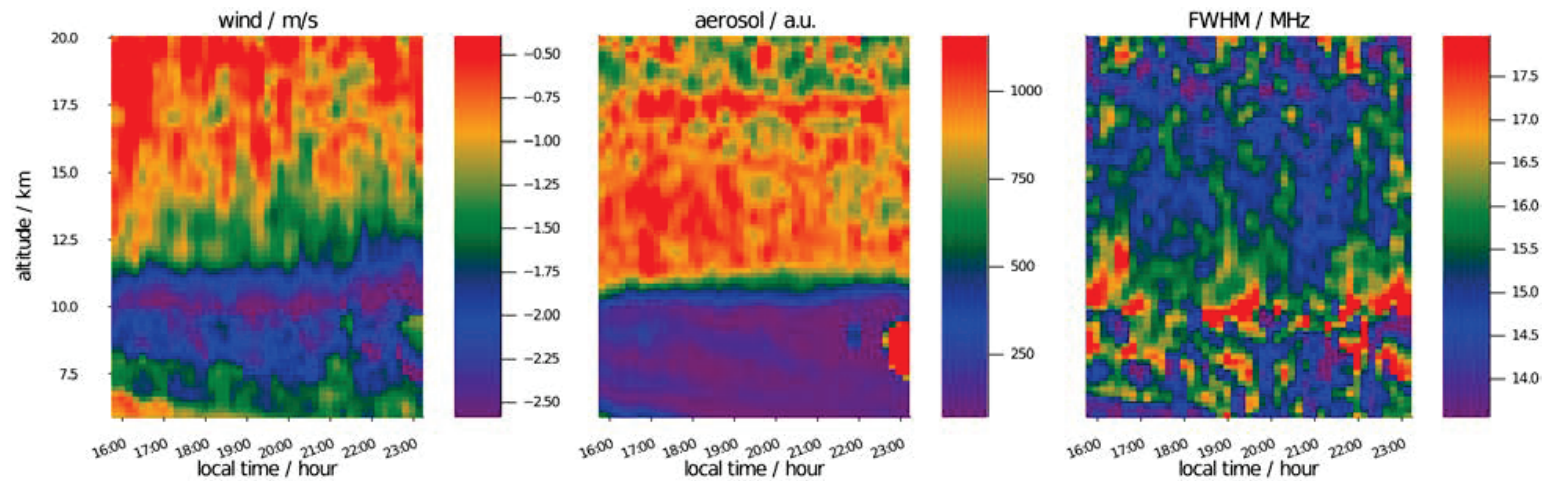

Figure 5: Horizontal Doppler wind, aerosol signal and spectral width measured by tilting the lidar approx. $2^{\circ}$ against the vertical, observing Doppler shifts of less than $2.5 \mathrm{~m} / \mathrm{s}$.

The main goal of this test measurement has been the matching of the spectral properties of laser and receiver and the capability to obtain high-precision spectra as discussed before in a compact setup with novel technology. Beside wind and aerosol, we also show the spectral width of the backscattered signal (Figure 5 right). In the stratosphere, the typical spectral width is in the order of $14.5 \mathrm{MHz}$. The convolution of the laser with the narrow bandwidth interferometer is $12.5 \mathrm{MHz}$. Therefore, the observed spectrums at these altitudes show a slight broadening compared to laboratory measurement. At lower altitudes with fast changing winds and a vertical gradient in the wind additional broadening occurs with values in the order of $18 \mathrm{MHz}$. Additional spectral broadening in the stratosphere should be caused by turbulent motions within the volume and would therefore open new measurement capability. In the future, the measurement of the spectral broadening 
as an indicator of turbulences will be investigated in more detail. The spectral broadening is also discussed more in detail in [9].

We designed VAHCOLI for polar regions and will install the network in the next years in north Scandinavia. Finally, such measurements will be obtained on a regular base within 20 fields of view, covering an area of more than $100,000 \mathrm{~km}^{2}$. The comparison with the former Fe-lidar shows that a 100 times larger signal is achievable with the next generation of the laser. By tilting $\sim 35^{\circ}$ a much larger Doppler effect is observable. In total, Doppler horizontal winds can be measured within a few seconds with this method by VAHCOLI. For space, we show simulations of the required signal strength in section 4. With this capability, VAHCOLI might be used in the future for calibration and verification of the next space mission Aeolus-FO. A network looking into different directions gives new insights about horizontal and also vertical structures since ground-based lidar with this technology achieve a much higher altitude resolution than possible from space.

\section{SPACEBORNE MULTI-FREQUENCY LIDAR FOR SIMULTANEOUS RAYLEIGH, MIE AND RESONANCE MEASUREMENTS}

\subsection{Doppler Rayleigh, Mie and resonance scattering}

Multi-frequency lidars are able to obtain Doppler measurements of temperature, wind and aerosol simultaneously. Depending on the scientific question, such an instrument is optimized to one or another scattering process or all at the same time. For simplification, we focus here on a very simple approach of obtaining the complete spectrum of Doppler Rayleigh, Doppler Mie or Doppler resonance scattering each. Our goal is an estimation what uncertainties are achievable if the system is optimized for a single scattering process only.

In Figure 6 we show the backscatter signal from Doppler iron (Fe: $386 \mathrm{~nm}$, metal density at the peak $\left.10,000 \mathrm{~cm}^{-3}\right)$, Doppler potassium (K: $770 \mathrm{~nm}$, metal density at the peak $25 \mathrm{~cm}^{-3}$ ), Doppler Rayleigh (386 nm, etalon with 2,000 MHz) and Doppler Mie $(386 \mathrm{~nm})$. For Mie we have assumed a broadening of a factor of 1.7 for the laser linewidth by SHG into the UV and a 1.7 times broader etalon compared to the measurement shown above in Figure 5. For Rayleigh and resonance scattering, the first three simulations assume $200 \mathrm{~K}$ and a signal of 100,000 counts if we tune the laser onto the maximum backscatter. After probing the spectrum with 21 frequencies, we obtain for a single spectrum the parameter given for an example on the left side. On the right side the uncertainties are obtained for 2,000 random simulations at constant temperature und $0 \mathrm{~m} / \mathrm{s}$ wind.

The uncertainty of the fit is given in the legend, followed by the rms of temperature or wind compared to the know temperature and wind. In practice only the uncertainties of the fit are available for real measurements. The rms shows that the uncertainties of the analysis are indeed in good agreement with the derived estimations of temperature and wind and serve as an independent control of the simulations. The last simulation assumes Mie scattering. Since the backscatter spectrum is much narrower, less signal is required to determinate the wind from the Doppler shift. Instead of 1,000 counts at the maximum of the backscatter, we obtain 520 counts by tuning the laser to the shown 21 frequencies. As a rule of thumb, approx. half signal strength is obtained by tuning the laser over one of the shown spectra in comparison of a laser tuned to the frequency with maximum backscatter. We will later simulate the signal from space for a laser operating on the maximum backscatter. For achieving the shown uncertainties, 100,000 counts must be obtained for Doppler resonance and Doppler Rayleigh. Only 1,000 counts are required for Doppler Mie if the laser is tuned to maximum backscatter only. The real count rate decreases by tuning the laser over the line as given in Figure 6 as real signal.

A comparison of iron with potassium shows that for the same signal iron achieves approx. 1.6 times smaller uncertainties for both, temperature and wind. Iron is therefore preferred if the same signal is achieved from space. The uncertainties for Doppler Rayleigh wind and temperature measurements are approx. 2 times larger compared to the peak of the iron layer. Even though we obtain only 520 photons by scanning the laser over the Mie peak, we can determinate wind with $10 \mathrm{~cm} / \mathrm{s}$ uncertainties from such a weak signal. A $1 \mathrm{~m} / \mathrm{s}$ uncertainty would require 100 times less signal if Gauss statistic could be assumed. However, such low statistic requires different calculations because of the low statistics in the wing of the line approaching zero counts. Since the Doppler shift at $386 \mathrm{~nm}$ for $1 \mathrm{~m} / \mathrm{s}$ is $5.4 \mathrm{MHz}$, the shown spectrum for Mie covers only a maximum line of sight wind of $\pm 5 \mathrm{~m} / \mathrm{s}$, which translate to approx. $\pm 10 \mathrm{~m} / \mathrm{s}$ horizontal wind. A 10 times larger range is required to cover a maximum Doppler shift range of $100 \mathrm{~m} / \mathrm{s}$. A lidar obtaining Doppler Rayleigh or Doppler resonance scattering simultaneously will cover this range anyways.

The method described here offers a simple solution for simultaneous Doppler measurements in flight direction and perpendicular. Different to a single frequency lidar the giant Doppler shift caused by the motion of the satellite is compensated by shifting the frequency of the laser according to the speed of the satellite. A straightforward solution is a separate seeder laser and switching the seeder laser if changing viewing direction. The technology described above does 
not require any stabilization of the seeder or pulsed laser to a fixed reference frequency. A single receiver is sufficient for all viewing direction with this straightforward approach. As mentioned in chapter 3, the ground-based lidar is currently extended to 5 fields of view switching between 5 mirrors between two laser pulses. We switch the viewing direction synchronized to the spectral measurement. After cycling multiple times over all frequencies, we chose the next direction depending on the scientific requirements.
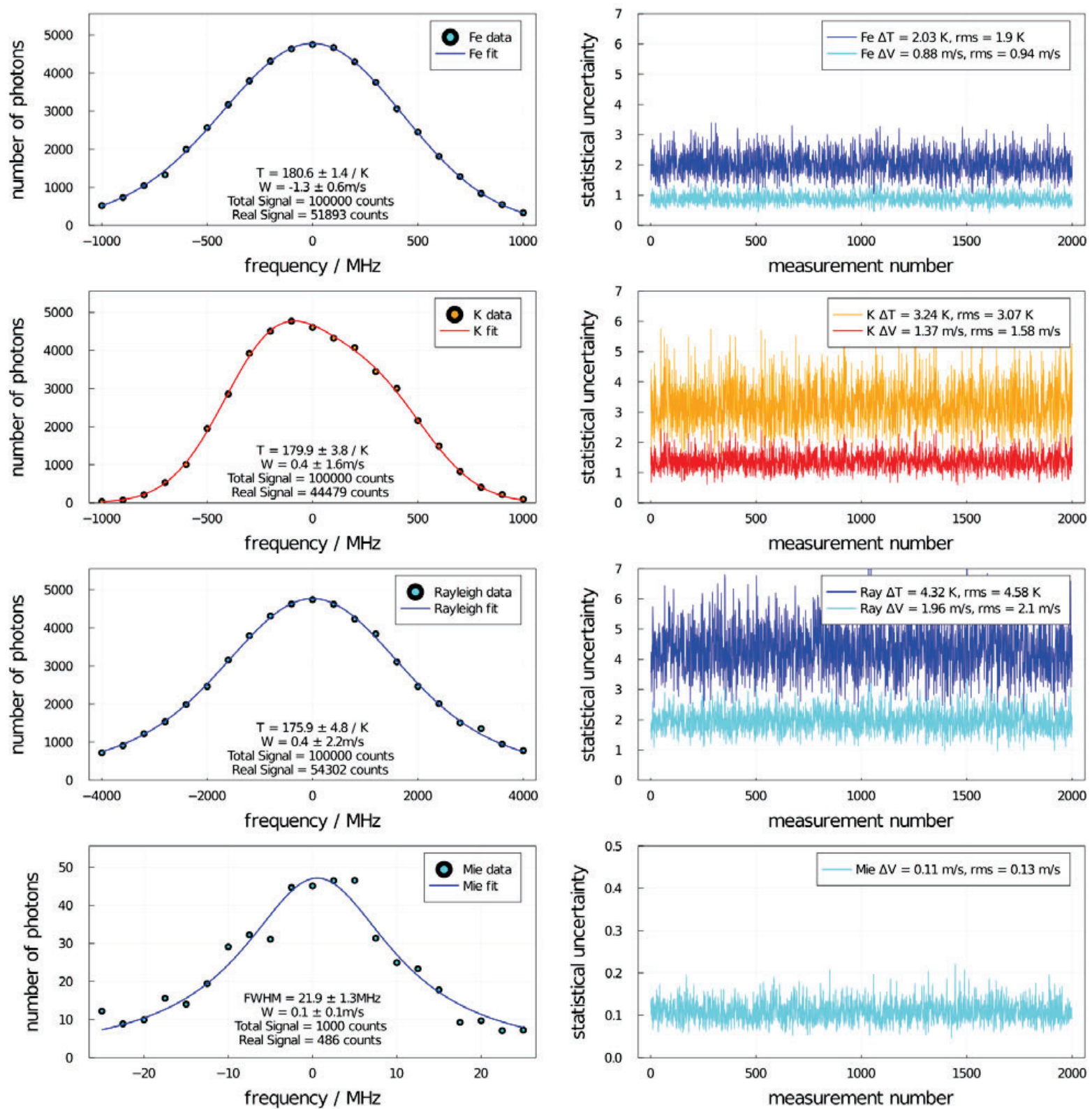

Figure 6: Left side: Simulated spectra of resonance scattering (Fe $386 \mathrm{~nm}$ and K $770 \mathrm{~nm}$ ), Rayleigh (behind $2000 \mathrm{MHz}$ etalon) and Mie (behind $16 \mathrm{MHz}$ etalon). Right side: Monte Carlo simulation for $200 \mathrm{~K}$ (resonance and Rayleigh scattering) and Mie. 
We note that the number of frequencies does not have any impact on the overall signal and uncertainties. Only a larger number of laser pulses is required to obtain a complete spectrum if we address more frequencies. With the alexandrite laser operating at $500 \mathrm{~Hz}$ much more frequencies are possible even if we must obtain the spectrum in a few seconds. For comparison, a single "observation" of Aeolus requires $12 \mathrm{sec}$ or 6000 laser pulses at its repetition rate of $50 \mathrm{~Hz}$. We also note again that an equal spacing of the frequencies is not required. Ground-based lidar use complex schemes. Since groundbased lidar control the frequencies by software, we achieve high flexibility.

\subsection{Signal from space}

Potassium $(770 \mathrm{~nm})$ and iron $(386 \mathrm{~nm})$ Doppler lidars are in use since 1995 by the IAP. Because of the better signal to background at daytime the IAP developed the mobile Fe lidar operating at $386 \mathrm{~nm}$ in 2002 [4][5]. Routine measurements of Doppler Fe, temperature and vertical wind at Davis, 69 S, Antarctica (2010-2012) [14] and Andenes (2014-2019), $69^{\circ} \mathrm{N}$ demonstrate the capability of such a system. The question remains if sufficient signal is obtained from space. For a simulation of the signal strength, we have chosen similar parameters compared to the Aeolus mission [15], namely a $30^{\circ}$ tilted $1.6 \mathrm{~m}$ telescope, an orbit of $320 \mathrm{~km}$ altitude, a measurement distance of $400 \mathrm{~km}$ and an overall transmission efficiency of the system of 0.3 . For comparison, Aeolus has achieved $\sim 100 \mathrm{~mJ}$ per pulse with a $50 \mathrm{~Hz}$ laser in the UV at $355 \mathrm{~nm}$, which correspond to an average power of $5 \mathrm{~W}$. For the simulation we have chosen $12 \mathrm{sec}$ which is the time for a single "observation" of Aeolus. We therefore assume a lidar of similar properties but with higher repetition rate laser and lower pulse energy resulting in the same average laser power.

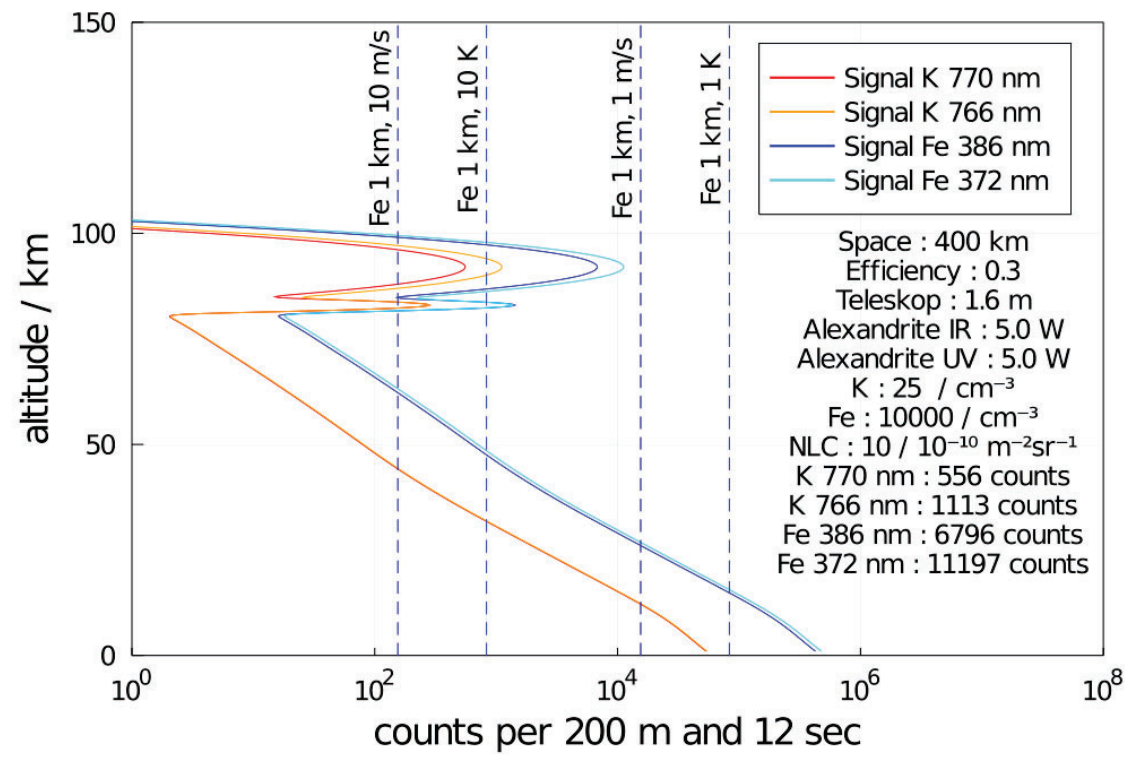

Figure 7: Simulation of the signal strength for a spaceborne lidar of similar properties compared to Aeolus. For space the potassium $(\mathrm{K})$ line at $766 \mathrm{~nm}$ or one of the iron lines are of interest. The difference between the two iron (Fe) lines is relatively small and the wavelength for a future space lidar depends therefore on the available optics and achievable efficiency. The metal layer between $\sim 80-100 \mathrm{~km}$ altitude is shown together with a Noctilucent cloud (NLC) at the lower boundary of the metal layer.

Figure 7 shows a simulation of the signal strength from space at four different wavelengths. The typical altitudes of metal layers are $80-100 \mathrm{~km}$ with a peak close to $90 \mathrm{~km}$. In summertime, ice particles (NLC) occur at polar regions at temperatures below about $145 \mathrm{~K}$ at a typical altitude of roughly $84 \mathrm{~km}$. Because of the high scientific relevance, we extended the simulation to demonstrate that these aerosols are also observable from space. Ground-based potassium lidar make use of the $770 \mathrm{~nm}$ because of strong absorption in the troposphere at the stronger line of $766 \mathrm{~nm}$. From space the IRwavelength of $766 \mathrm{~nm}$ allows Doppler Mie and resonance measurements with the disadvantages of higher solar background compared to iron, but the advantage of a relatively simple approach compared to a lidar operating in the UV. As we will 
discuss later the background is nevertheless in all cases lower compared to Aeolus. In particular, Doppler Mie is observable with orders of magnitude lower background compared to Aeolus and therefore undisturbed by the solar background at day for all cases. The real challenge is resonance scattering at daytime because of the atomic physics involved. Since the signal of iron is much larger and the simulations before show that the uncertainties for temperature and wind are smaller at the same count rate, iron is the preferred solution for the mesosphere independent on the chosen wavelength (386 $\mathrm{nm}$ or $372 \mathrm{~nm})$.

Since measurements from space must be fast, we have chosen as mentioned before $12 \mathrm{sec}$ integration time similar to Aeolus but with $200 \mathrm{~m}$ altitude resolution. From the simulation, it is clear that the metal layer is observable with even shorter time resolution and/or higher altitude resolution. However, Doppler measurements require a larger signal strength. From the simulation before we can draw lines equivalent to different temperature and wind uncertainties. With a laser of $5 \mathrm{~W}$ operating in the UV, uncertainties for wind approach $1 \mathrm{~m} / \mathrm{s}$ at the peak with $1 \mathrm{~km}$ resolution. $10 \mathrm{~m} / \mathrm{s}$ is achieved over a large part of the iron layer. The uncertainties for Doppler resonance temperature are larger but still in a reasonable range. We note that waves in the mesosphere can easily be as large as $\pm 25 \mathrm{~K}$. The horizontal wind exceeds $100 \mathrm{~m} / \mathrm{s}$ in this altitude range.

The achievable signal supports therefore observations of the mean state, gravity waves and tides and with even higher resolution the metal layer as tracer for waves, or e.g. sporadic layers observed at the upper part of the metal layer [16]. Sporadic layers are a link to the ionized thermosphere and frequently observed by ground-based lidar. NLC are also observable from space extending the capability again. In comparison with Aeolus, we achieve similar uncertainties for wind in the order of a few $\mathrm{m} / \mathrm{s}$ over a large part of the metal layer. In contrast to potassium and sodium, which show a semiannual variation of the density, iron benefits from an all-season and global wide relatively constant metal layer with number densities of approx. $10,000 \mathrm{~cm}^{-3}$ at the peak of the layer. The simulations shown here reflect therefore average global-wide conditions, whereas for sodium and potassium the mean density drops by one order of magnitude or more over the season and the solar background is a major limitation not existing for iron as we will show next. An iron lidar has therefore a much more uniform performance global-wide.

\subsection{Solar background}

Ground-based and spaceborne lidar must obtain measurements at daytime. Ground-based resonance lidars use narrow bandwidth filtering in the order of the atomic linewidth since decades to enable measurements at day [5]. In contrast, Aeolus reduces the solar background with a small field of view only. Aeolus has a $1 \mathrm{~nm}$-broad interference filter followed by low finesse interferometers. In contrast, ground-based lidars use the combination of narrow field of view and narrow bandwidth spectral filtering in the order of a few picometer by high finesse interferometer or special filter such as Faraday filter (potassium and sodium). A resonance lidar benefits additionally from a deep Fraunhofer line. Such lines are only roughly $10 \mathrm{pm}$ broad. Because of the multi-frequency capability, the same interferometer for Doppler measurements acts additionally as a filter suppressing the solar background. Similar to Aeolus only two interferometers and interference filters are required. We can compare the solar background of Aeolus and an iron lidar in space with narrow bandwidth filter by considering the solar spectrum and calculating the impact of narrow bandwidth filtering on the solar spectrum. Systems with otherwise identical parameter are comparable in that way.

Figure 8 shows on the left side the solar background with high resolution. Fraunhofer lines are only visible as vertical lines here. We have indicated the wavelengths of Aeolus, iron (Fe, $386 \mathrm{~nm})$ and potassium (K, $770 \mathrm{~nm})$ which are in use by the ground-based lidar of the IAP. At the right side we show the spectrum of $\pm 0.5 \mathrm{~nm}$ which correspond to the FWHM of the interference filters of Aeolus. More detailed calculations considering the additional blocking of the two interferometers of Aeolus, as far as we know from literature [15], show a further reduction of the solar background by a factor of 9 (Rayleigh) and 4.5 (Mie). Otherwise, the solar background is given by the red spectrum times the interference filter. For iron narrow bandwidth filtering can reduce the background by magnitudes if efficient blocking over the whole visible range is achieved. For iron narrow bandwidth filters in the order of $1 \mathrm{pm}$ can be applied.

The theoretical limit for Rayleigh and resonance scattering is a 2,000 times lower solar background compared to Aeolus even if we consider the additional blocking of the two interferometers of Aeolus. For Mie scattering spectral filters in the order of the laser linewidth can be deployed. For the alexandrite laser with a linewidth of a few MHz it is possible to suppress the solar background by 500,000 compared to the Aeolus Mie channel. Such narrow bandwidth filters are in regular use for ground-based lidars and have been the key development for achieving observations at day in the mesopause region. However, different to Rayleigh and Mie scattering the field of view of a resonance lidar depends on the atomic physics. For sodium and potassium, the field of view must be large due to saturation effects and only a poor signal to 
background ratio of $\sim 1$ is achieved at daytime. With the development of the Doppler iron lidar, the IAP has demonstrated that this is not the case for iron.
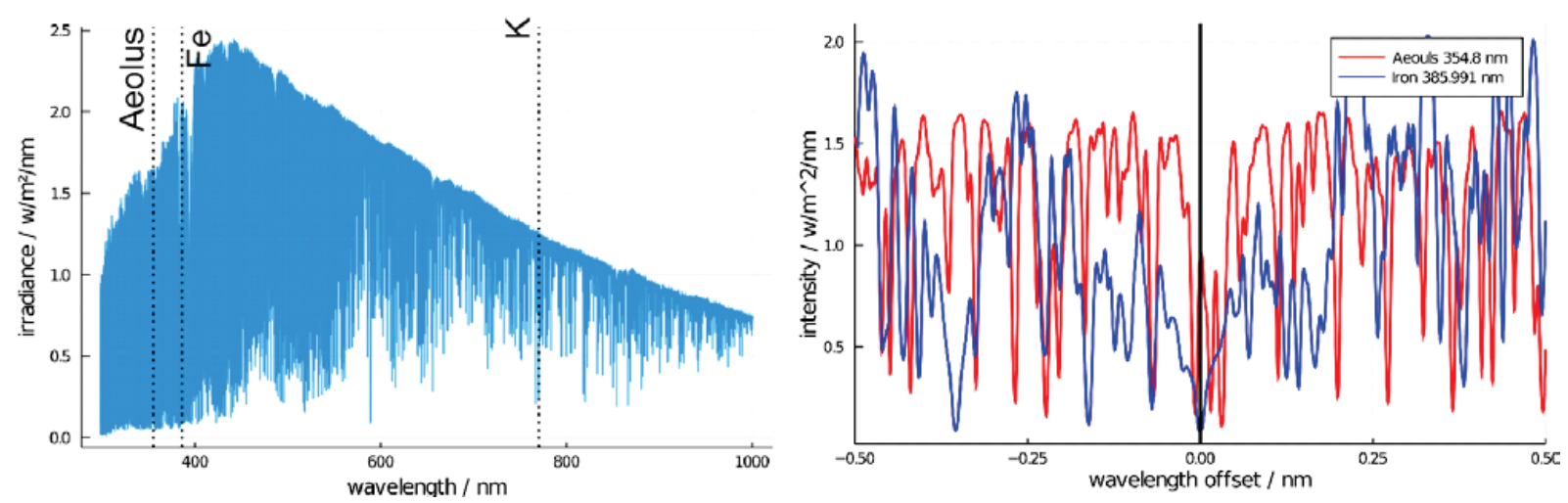

Figure 8: Left side: Solar spectrum with high resolution. Fraunhofer lines are visible as vertical lines. Right side: Solar spectrum within the FWHM of a $1 \mathrm{~nm}$ filter for the wavelength of Aeolus and Fe $386 \mathrm{~nm}$. The product of filter transmission and solar spectrum gives the solar background. With narrow bandwidth spectral filtering the background is given at a single point at the center wavelength. The vertical black line indicates the filter width of a narrow bandwidth filter in comparison to the $1 \mathrm{~nm}$ interference filter of Aeolus.

As an example of such a measurement, Figure 9 shows a raw data signal of the flashlamp-pumped alexandrite laser based Doppler Fe-lidar of the IAP. As discussed before, Doppler Mie observations at daylight are only limited by the detector dark count rate. The mobile lidar performs Doppler Mie measurements at the fundamental wavelength of the laser at $772 \mathrm{~nm}$ (orange channel) in the mesosphere with a narrow bandwidth filter of $25 \mathrm{MHz}$ (50 fm). The shown signal are raw data integrated over all frequencies without subtracting any background. Since the dark count rate of a good detector is in the order of 20 counts per sec, the average background of this channel is below one count per 200 m altitude resolution after integrating the signal about 2 min (4,000 laser pulses). For this channel, we have indicated the typical Rayleigh signal of this channel as straight line to guide the eye.

The blue channel shows the resonance scattering in the mesopause region at day. Since the spectral width of resonance scattering is much broader, a remaining background is still visible. We note that optimal spectral filtering is not achieved with this old system. Theoretical calculations show that we can reduce nowadays the solar background by a factor 14 with an advanced interferometer. This old system operated at a field of view of $65 \mu \mathrm{rad}$ with an $80 \mathrm{~cm}$ telescope compared to Aeolus with $20 \mu \mathrm{rad}$ field of view and $1.6 \mathrm{~m}$ telescope. With the parameters of Aeolus the background would decrease by a factor of approx. 10 by reducing the field of view. On the other side, the larger mirror increases the background by a factor of 4. In summary, the background in space with optimal spectral filtering and larger mirror and field of view of $20 \mu \mathrm{rad}$ would be roughly 35 times lower and approaching the dark count rate of the detector. This example and theoretical calculations show, that nearly solar-blind observations with a dark count rate of roughly $20 \mathrm{~Hz}$ is achievable from ground and space with nowadays technology at the iron line.

A multi-frequency lidar can achieve already a high performance for Doppler measurements with very low solar background and two interferometers only. Optimal spectral filtering, however, requires an additional interferometer. We apply this technology for VAHCOLI at $770 \mathrm{~nm}$. An iron lidar has a better signal to background at day and total signal but requires a more complex technology. The goal of VAHCOLI are reliable, compact and cost-efficient systems for a network of Doppler lidars. For such a network, a potassium lidar at $770 \mathrm{~nm}$ has the combination of high performance at low cost and complexity. Nevertheless, they can be developed further into a Doppler iron lidar network by extending the technology similar to the existing mobile Fe-lidar. The IAP has operated such a system at various locations over many years with success. The technology is therefore field-proven even though based so far on 25-year-old flashlamp-pumped laser technology not suitable for space. With the development of the diode-pumped alexandrite laser this comes within reach only now. We also note again that iron is the optimal wavelength for a Doppler resonance lidar operating at day whereas Doppler Mie is already measured background free by the Fe-lidar at the $772 \mathrm{~nm}$ line. With this technology, Doppler Mie is achievable from space without second harmonic generation in the infrared as shown for VAHCOLI before. 


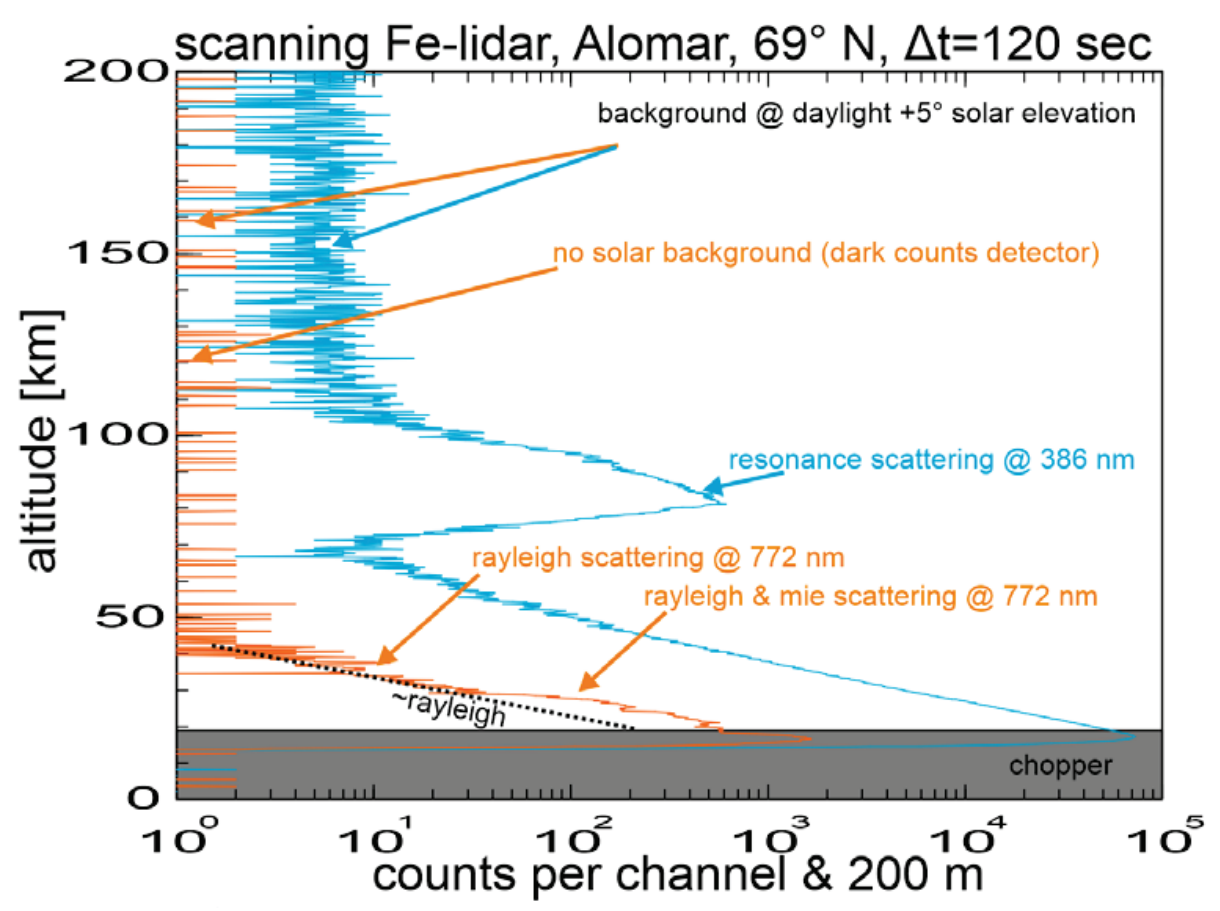

Figure 9: Exemplary measurement of the mobile iron lidar with a flashlamp-pumped alexandrite laser for $386 \mathrm{~nm}$ (blue) and $772 \mathrm{~nm}$ (orange). A double etalon suppresses the solar background at $386 \mathrm{~nm}$. A triple etalon with a narrow bandwidth filter of $50 \mathrm{fm}$ is used for Doppler aerosol measurements (orange channel).

\section{ROADMAP TO A SPACEBORNE MULTI-FREQUENCY LIDAR}

The lidar proposed in the former section bases on the principle setup of Aeolus concerning the satellite platform and the mono telescope. Also, there are many similarities in the principle optical setup, such as the monostatic telescope and the direct measurement of the emitted laser in the same detector path. The mentioned narrow bandwidth filters are comparable in the complexity of the Aeolus interferometer and easy to adapt. Therefore, the main challenge is the emitter based on a frequency doubled diode-pumped alexandrite laser instead of the frequency-tripled diode-pumped Nd:YAG laser used for Aeolus.

In former publications [7][17][18] the suitability of the laser technology with a diode-pumped alexandrite laser was investigated. The baseline for a spaceborne laser technology is the mounting technology, developed at the Fraunhofer ILT and Airbus Defense \& Space with the support of DLR and ESA, where the optics are directly soldered without the use of any organics. It was demonstrated in "FULAS (Future Laser System) platform" [19] and used for the laser emitter for MERLIN, a French-German space lidar mission dedicated to atmospheric methane [20] that is also presented at the ICSO2020 [21].

For spaceborne operation of the laser system the optical elements have to be qualified regarding operation in low earth orbit. For most of the components necessary for the diode-pumped alexandrite laser, e.g. mirrors, lenses, Faraday rotator, Pockels cell, there are already technological solutions developed and qualified [22]. However, no publications on radiation testing were found for the pump diode emitter material and the alexandrite crystal, so space qualification of the pump diodes and the laser medium is still pending.

For the evaluation of the laser design regarding stability under space condition, a performance analysis was carried out. Regarding the stability against tilting, the mirrors of the resonator are by far the hardest to fulfill specification. Despite the tentative mechanical setup and the non-optimized folding for a small footprint, the results indicated that the mirror mounts developed at ILT for spaceborne lasers with tilting $<10 \mu \mathrm{rad}$ will suffice to set up the resonator.

The pulse energy that is necessary for a high temporal and spatial resolution strongly depends on the mission parameters. With an average power of $\sim 5 \mathrm{~W}$ at the UV-wavelength within the Fraunhofer line, an integration time of $12 \mathrm{~s}$ would provide an altitude resolution of $1 \mathrm{~km}$ and with an orbit similar to Aeolus, leading to a horizontal integration of 
$90 \mathrm{~km}$. With repetition rates of $500 \mathrm{~Hz}$ and $1 \mathrm{kHz}$, that are both well suited for the data acquisition and processing, the pulse energy at the dedicated wavelength has to be $>10 \mathrm{~mJ}$ and $>5 \mathrm{~mJ}$ respectively.

\subsection{Energy scaling for spaceborne measurements}

Energy scaling can be achieved with a higher level of pump power of the oscillator or with a master oscillator with successive power amplifier (MOPA) setup. The advantage of a MOPA system is the control of the spectral and spatial properties in an oscillator with lower thermal and optical load on the components and a separated power amplification with little influence on the latter properties. First attempts for an amplifier based on diode-pumped alexandrite are reported [23], showing the possibility for an energy-scaling, though suffering from significant loss of beam quality.

With the InnoSlab amplifier concept [24], the FULAS platform involves a technology for energy scaling, that has proven to ensure high efficiency and keeps the spectral and especially spatial properties almost unchanged for Nd:YAG [19][25] and can be adapted to diode-pumped alexandrite. Nevertheless, an additional amplifier stage will increase the complexity.

As the pump power of the current oscillator is rather moderate, the approach of higher pump power in the oscillator is worth investigation. Therefore, a new pump unit with up to $400 \mathrm{~W}$ peak power, which is 2.67 times higher than the current unit, was developed. The pump light is delivered from a fiber with $800 \mu \mathrm{m}$ core diameter and a Numerical Aperture of 0.22 , resulting in a beam quality of $\mathrm{M}^{2}=430$, as shown in Figure 10 .
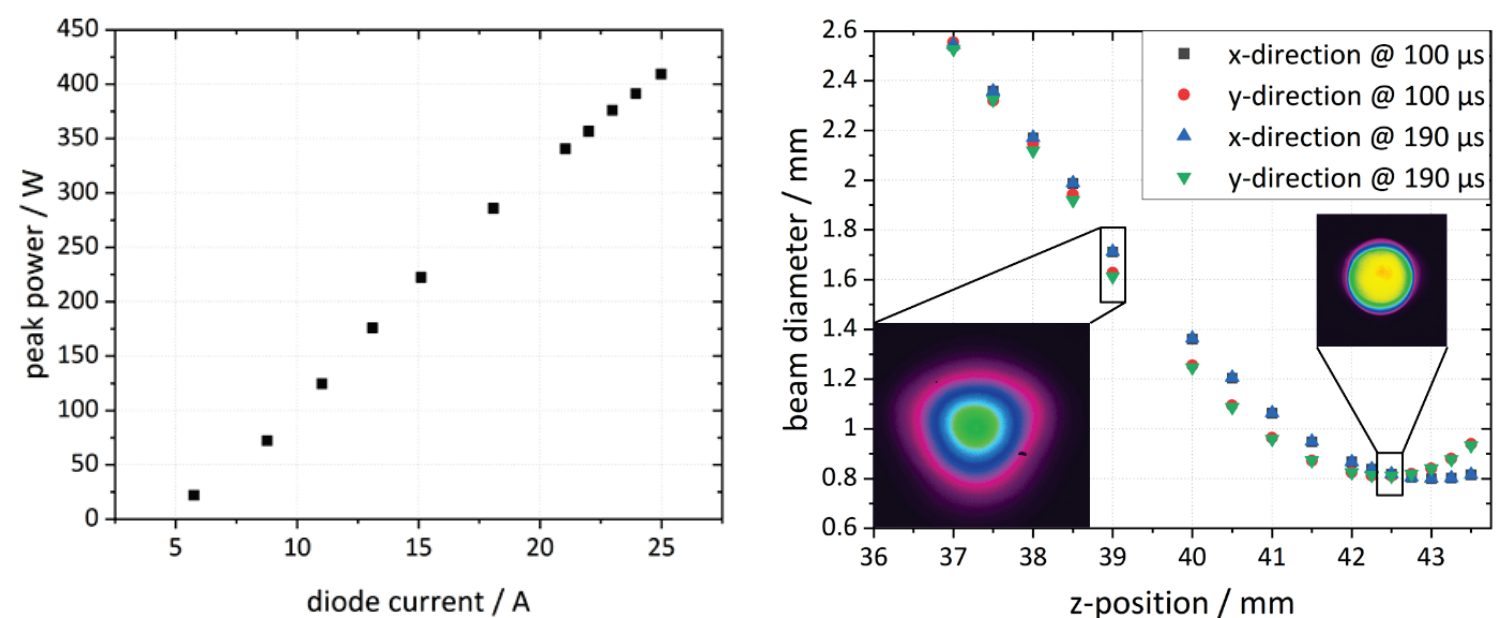

Figure 10: Output peak power in dependence of the current and caustic with beam profile for different pump pulses of the new pump unit.

The radiance (or brightness) is reduced by a factor of 1.5 compared to the current pump unit, but it is still possible to generate a caustic in the crystal that keeps the pump energy density unchanged. Therefore, a focus diameter of $400 \mu \mathrm{m}$ is generated $2 \mathrm{~mm}$ behind the input facet of the $7 \mathrm{~mm}$ long alexandrite crystal with $0.2 \mathrm{at} \%$ doping, leading to an effective pump diameter, that is $\sqrt{ } 2.67=1.63$ times larger compared to the current laser of VAHCOLI. As the other optical components in the energy-scaled oscillator are the same as in the current one, the resonator losses are unchanged as well. By designing the resonator in a way to fit the resonator mode to the given effective pump beam size, the working point of the oscillator is unchanged, and its performance should also be unchanged, except of the output energy.

The first laboratory setup of the ring-resonator yields an unidirectional output pulse energy of up to $4.6 \mathrm{~mJ}$ at the potassium line $(770 \mathrm{~nm})$ with a beam quality of $\mathrm{M}^{2}=1.1$, shown in Figure 11. The oscillator is operated in a seeded Qswitch operation at a repetition rate of $500 \mathrm{~Hz}$ with a pulse duration of $800 \mathrm{~ns}$. Due to the large laser mode and the strong thermal lens induced by the increased pump energy, the energy-scaled resonator has a smaller stability region compared to the former resonator. This is the reason for the sudden drop for pump energies below $30 \mathrm{~mJ}$ and again below $22 \mathrm{~mJ}$ in the output energy in dependence of the pump energy shown in Figure 11. That is also the reason why the pump energy is still reduced as the resonator in its current design does not allow larger thermal lenses. By changing the concave mirrors to compensate for stronger thermal lenses, the pump energy and thereby the output energy can be scaled further.

The exact linewidth could not be measured, as the spectrometer is not sufficiently sensitive. As the oscillator operates at the same working point as the ones in [9] and the cavity control technique is the same, there is no obvious reason, why 
the linewidth should be larger. It will be controlled, once the laser is integrated into the lidar system, where a narrow bandwidth etalon can be used to measure linewidths below $10 \mathrm{MHz}$.

As the setup is only a first test design and rather tentative, a careful resonator design with a small footprint is planned to be integrated in the available space in the lidar. With the data on the thermal lens in the crystal, caused by the new pump unit, and careful adjustment of the laser mode radius inside the crystal, the efficiency and thereby the output energy is expected to be raised as well.
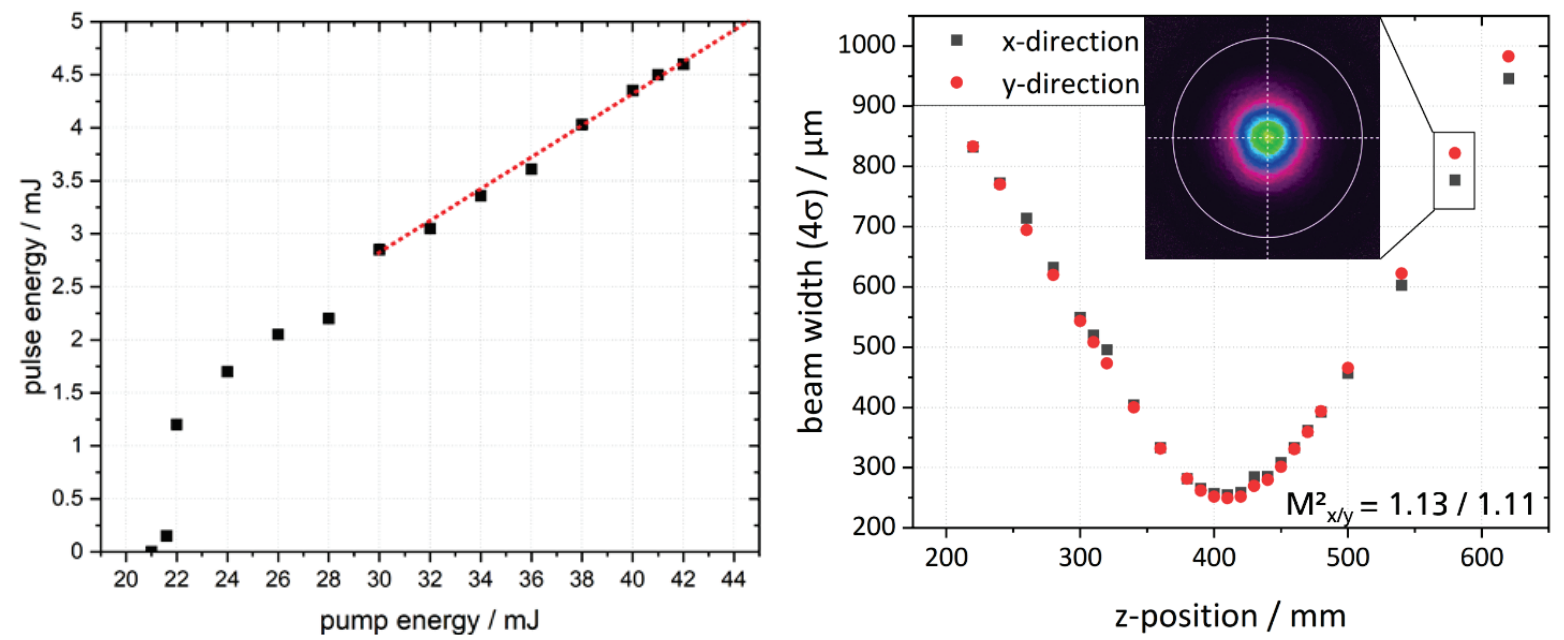

Figure 11: Output energy (with slightly reduced pump energy) and caustic of the energy-scaled oscillator in seeded Qswitched operating at $500 \mathrm{~Hz}$ repetition rate.

Based on the new optical design of the power-scaled pump unit, a version with an output pump peak power of $800 \mathrm{~W}$ from a fiber with $1,000 \mu \mathrm{m}$ core diameter and a Numerical Aperture of 0.22 is planned. This pump unit would have a higher radiance and with a suitable resonator design, the working point can again be maintained, yielding output energies up to $10 \mathrm{~mJ}$ while preserving the spatial and spectral properties.

It is noted that the diodes are capable of duty-cycle up to $10 \%$ with the stated peak power, but for the current laser with $500 \mathrm{~Hz}$ repetition rate and $100 \mu$ s pump duration, only $5 \%$ is used. The repetition rate of the laser can be raised with only slight adaptions due to the higher average heat deposition in the crystal.

These first results and the prospect of a straightforward energy-scaling of the oscillator and the availability of a suitable amplifier technology show that a diode-pumped alexandrite laser can fulfill the energetic specifications of a future spaceborne mission.

\subsection{Frequency-conversion to address Fraunhofer-line}

The field campaigns as the demonstration of the compact Doppler lidar based on diode-pumped alexandrite laser were conducted at the resonance of potassium at $770 \mathrm{~nm}$ with the fundamental wavelength of the laser. For a lidar that can operate free of solar underground, a deeper Fraunhofer line as for the iron resonance lines at $386 \mathrm{~nm}$ or $372 \mathrm{~nm}$ in the UV is better suited. Both wavelengths would contribute to the eye-safety aspects of such a mission. Which of both wavelengths is better suited for such a lidar has to be analyzed in detail, as there are pros and cons for each of them. Nevertheless, both iron resonance lines can be addressed with a single-staged frequency-doubling of the diode-pumped alexandrite laser, reducing the complexity compared to the two-staged frequency-tripling, necessary for Nd:YAG Master Oscillator Power Amplifier (MOPA) lasers [26].

The fundamental wavelength for the line at $386 \mathrm{~nm}$, namely $772 \mathrm{~nm}$, is only $2 \mathrm{~nm}$ separated from the current wavelength for potassium. The resonator does not have to be modified to operate at the new fundamental wavelength and the performance does not change. Such a system was demonstrated for flashlamp-pumped alexandrite lasers with high conversion efficiencies up to $61 \%$ [4].

For the frequency-doubling, two concepts, extra- or intra-cavity, are possible as long as a single oscillator solution without amplifier is chosen. The extra-cavity conversion with a non-linear crystal subsequent to the oscillator is less 
complex as the laser resonator and conversion process are completely separated. To provide a high fluence within the nonlinear crystal, ensuring a high conversion-efficiency, the laser beam is focused to a small diameter.

By contrast, for the intra-cavity conversion, the non-linear crystal is placed within the resonator, benefitting from the enhanced intra-cavity energy. The cavity-enhancement is given by the resonator losses and the outcoupling. The converted energy is coupled out of the resonator at the next resonator mirror and represents the total outcoupling of the resonator as there is no outcoupling of the fundamental wavelength. The conversion efficiency has to be optimized for an efficient and stable laser operation by tuning it to the optimal outcoupling degree for the fundamental laser. For the given resonators with very low losses, outcoupling mirrors and thereby conversion efficiencies of $\sim 3 \%$ are optimal and allow for very moderate optical load for the non-linear crystals. As the laser is cavity-controlled and q-switched with ns-pulses, a feedback on the laser process, such as "green-noise" issues, known from laser in cw operation, does not occur.

Using the proprietary software package OPT [27], developed at ILT, both conversion concepts, extra- and intra-cavity, are investigated numerically and evaluated. For different crystal lengths, the conversion efficiency is shown for different intensities see Figure 12. For the extra-cavity conversion, theoretical efficiencies over $80 \%$ are possible, leading to pulse energies up to $4 \mathrm{~mJ}$ at the iron resonance line for an assumed energy-scaled laser with $5 \mathrm{~mJ}$ at the fundamental wavelength and a diameter of $\sim 200 \mu \mathrm{m}$ in the non-linear crystal.

For the intra-cavity conversion, the non-linear crystal is placed within the cavity at a position where the laser mode has a diameter of $\sim 800 \mu \mathrm{m}$. The outcoupled energies would be directly at the UV wavelength even though the conversion efficiency in respect to the intra-cavity energy is only $3 \%$ (green line). But the light at the fundamental wavelength that is not converted stays in the cavity and contributes to the laser process at the fundamental wavelength. The different envisaged outcoupled energies are $2 \mathrm{~mJ}$ for the laser operating currently in VAHCOLI, $5 \mathrm{~mJ}$ for the assumed output of the setup currently developed in the lab and $10 \mathrm{~mJ}$ for the planed further energy-scaled laser with the planed diode module.

The envisaged crystal lengths are available off-the-shelves in good quality, and the intensities are well below any Laser Induced Damage Thresholds (LIDT) of the coatings. The experimentally realization of both concepts is planned for the near future.
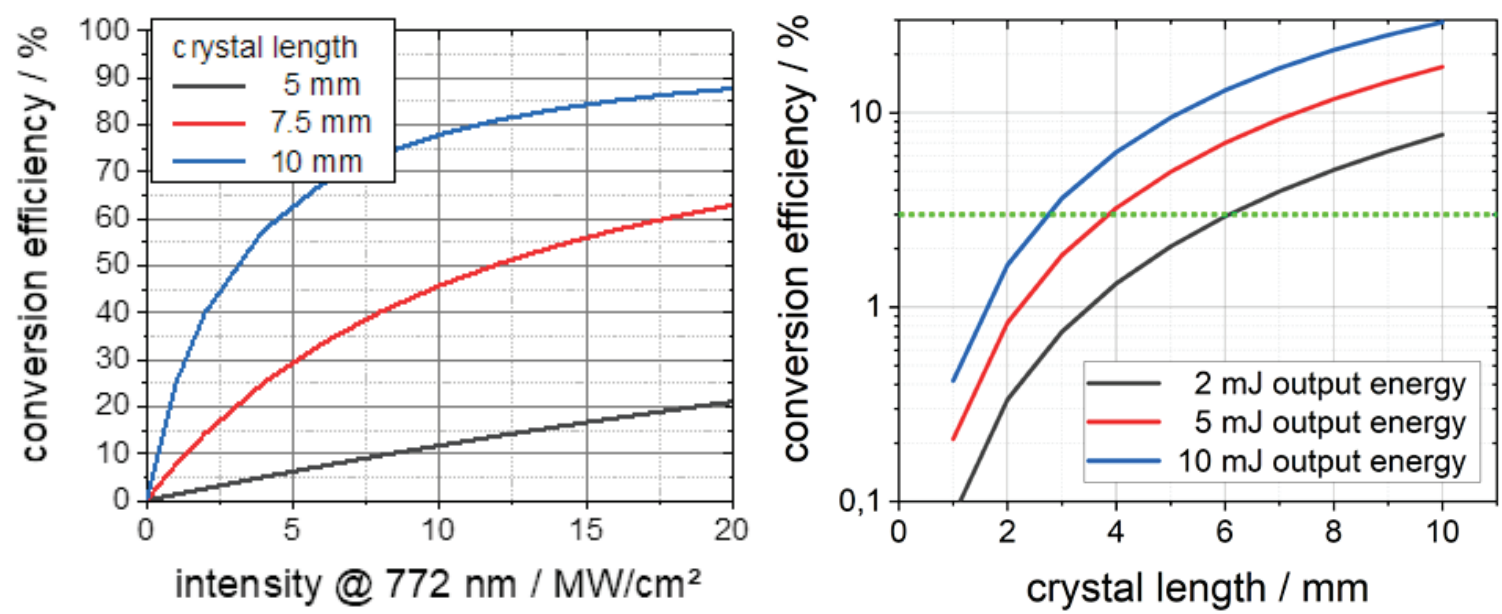

Figure 12: Efficiency of extra-cavity conversion for different non-linear crystal lengths (left) and the optimal crystal length for intra-cavity conversion to ensure the optimal outcoupling of $\sim 3 \%$ (right) with a laser operating at the fundamental wavelength of $772 \mathrm{~nm}$.

\section{CONCLUSION}

We have shown that a network of ground-based lidar multi-frequency lidar is under construction, based on diode-pumped alexandrite lasers. With a size of $1 \mathrm{~m} \times 1 \mathrm{~m} \times 1 \mathrm{~m}$ the prototype of such a system demonstrates already Doppler aerosol measurements out of a compact, automatic system. Built from off-the-shelf components, including a PC, such a system requires $500 \mathrm{~W}$ electrical power at a weight of less than $500 \mathrm{~kg}$. Since PC and housekeeping requires more than half of the electrical power it is clear that such a system fits in size, weight and electrical power to the limitations of space lidar even though build as a ground-based lidar. 
Key features are the novel laser technology and multi-frequency capability with a receiver efficiently blocking the solar background and allowing at the same time Doppler resonance, Doppler Rayleigh and Doppler Mie measurements with two detectors and two interferometers. We have shown in a first field campaign that the spectral properties of laser and lidar allow Doppler Mie wind measurements even though the system is to a larger degree 3D-printed. Equipped with a novel advanced ramp and fire technology we control the frequency of the laser with sub-MHz resolution from pulse to pulse. A linewidth of $3.3 \mathrm{MHz}$ translates to $\sim 6 \mathrm{MHz}$ at a UV wavelength, compared to $50 \mathrm{MHz}$ of Aeolus.

A multi-frequencies lidar offers a simple solution for simultaneous measurements of the Doppler shift in flight direction and perpendicular by switching to a second seeder laser at the same time we switch the viewing direction. Because of the giant Doppler shift in flight direction a single seeder laser might not be able to compensate the Doppler shift caused by the motion of the satellite in the short time the viewing direction is switched. Switching to a second seeder offers a straightforward solution whereas the flexibility of a multi-frequency lidar compensates for changing Doppler shifts caused by a change in the speed caused by the orbit.

We have shown in simulations that solar-blind Doppler Mie measurements are achievable at all wavelength. Observations of Doppler Rayleigh and resonance are nearly solar-blind for iron, with orders of magnitude lower solar background compared to Aeolus or existing potassium and sodium resonance lidars. We have shown that iron is the preferable solution for a resonance lidar in space, observing also the mesosphere with similar capability for wind and temperature as Aeolus achieves in the troposphere and stratosphere. The metal layer is observable from space with even higher altitude and time resolution. Iron densities benefit also from a relative constant metal layer worldwide whereas sodium and potassium show large variation above the globe.

We have estimated the signal strength of a spaceborne lidar with similar properties compared to the ADM-Aeolus mission. By combining high repetition rate with low pulse energy, a ring laser with intra- or extra-cavity frequency conversion can achieve the required laser power without any amplification stages and therefore less complex laser technology. The efficiency of the laser is twice as high and allows therefore double the laser power on the same satellite platform. Theoretical calculation of the signal strength, solar background and signal required for Doppler measurements show a few m/s or K is achievable from space for an iron lidar operating at $386 \mathrm{~nm}$ or $372 \mathrm{~nm}$ with $5 \mathrm{~W}$ average power in the UV. By applying narrow bandwidth spectral filter within a deep Fraunhofer line such a lidar is almost solar-blind and limited only by the dark count of the detector. Observations by a mobile ground-based lidar prove the concept of a multifrequency lidar since many years.

With the development of a diode-pumped alexandrite laser, it now becomes possible to apply this technology for future space mission, extending the capability of Aeolus to the mesosphere with a single mission. We showed the current development on the roadmap to a spaceborne lidar based on a diode-pumped alexandrite laser fully in line with the mature FULAS platform.

\section{REFERENCES}

[1] https://www.esa.int/Applications/Observing the Earth/Aeolus

[2] Solomon, S. C., Liu, H.-L., Marsh, D. R., McInerney, J. M., Qian, L., and Vitt, F. M., "Whole atmosphere simulation of anthropogenic climate change," Geophys. Res. Lett. 45, 1567-1576 (2018).

[3] Akmaev, R. A., "On estimation and attribution of long-term temperature trends in the thermosphere," J. Geophys. Res., 117, A09321 (2012).

[4] Lautenbach, J. and Höffner, J., "Scanning iron temperature lidar for mesopause temperature observation," Appl. Opt., 43(23), 4559-4563 (2004).

[5] Höffner, J. and Lautenbach, J., "Daylight measurements of mesopause temperature and vertical wind with the mobile scanning iron Lidar,” Opt. Lett., 34, 1351-1353 (2009).

[6] Munk, A., Strotkamp, M., Walochnik, M., Jungbluth, B., Traub, M., Hoffmann, H.-D., Poprawe, R., Höffner, J. and Lübken, F.-J., "Diode-pumped Q-switched Alexandrite laser in single longitudinal mode operation with Watt-level output power," Opt. Lett. 43, 5492-5495 (2018).

[7] Strotkamp, M., Munk, A., Jungbluth, B, and Höffner, J., "Diode-pumped Alexandrite laser for next generation satellite-based earth observation lidar," CEAS Space J 11, 413-422 (2019).

[8] Lübken, F.-J. and Höffner, J., "VAHCOLI, a new concept for lidars: technical setup, science applications, and first measurements," Atmos. Meas. Tech. Discuss. [preprint], https://doi.org/10.5194/amt-2021-33, in review, (2021). 
[9] Munk, A., Strotkamp, M., Jungbluth, B., Froh, J., Mense, T., Mauer, A., Höffner, J., "Rugged diode-pumped Alexandrite laser as an emitter in a compact mobile lidar system for atmospheric measurements," Appl. Opt., submitted (2021).

[10] von Zahn, U., Höffner, J., Eska, V., and Alpers, M., “The mesopause altitude: Only two distinctive levels worldwide?”, Geophys. Res. Lett. 23, 3231-3234 (1996).

[11] von Zahn, U. and Höffner, J., “Mesopause temperature profiling by potassium lidar,” Geophys. Res. Lett. 23, 141-144 (1996).

[12] Munk, A., Jungbluth, B., Strotkamp, M., Hoffmann, H.-D., Poprawe, R., and Höffner, J., "Alexandrite laser in Q-switched single longitudinal mode operation pumped by a fiber-coupled diode module," Proc. SPIE 10896, 1089610 (2019).

[13] Munk, A., Jungbluth, B., Strotkamp, M., Hoffmann, H.-D., Poprawe, R., Höffner, J. and Lübken, F.-J., "Diodepumped alexandrite ring laser in single-longitudinal mode operation for atmospheric lidar measurements," Opt. Express 26, 14928-14935 (2018).

[14]Lübken, F.-J., Höffner, J., Viehl, T. P., Becker, E., Latteck, R., Kaifler, B., Murphy, D. and Morris, R.J., "Winter/summer transition in the Antarctic mesopause region," J. Geophys. Res. Atmos., 120(12), 394-12,409 (2015).

[15] Reitebuch, O., Huber, D., and Nikolaus, I.: “ADM-Aeolus Algorithm Theoretical Basis Document (ATBD) Level-1B Products”, AE-RP-DLR-L1B-001, v. 4.4, 117 pp. (2018).

[16] Alpers, M., Höffner, J., and von Zahn, U., "Iron atom densities in the polar mesosphere from lidar observations," Geophys. Res.Lett., 17, 2345-2348 (1990).

[17] Strotkamp, M., Munk, A., Jungbluth, B, Hoffmann, H.-D., and Höffner, J., "Diode-pumped Alexandrite laser instrument for next generation satellite-based Earth observation," Proc. SPIE 11180, 111800F (2019).

[18] Höffner, J., Strotkamp, M., Munk, A., Jungbluth, B., "Demonstration of a compact and universal Doppler lidar based on a novel diode pumped alexandrite ring laser," Proc. SPIE 11180, 1118029 (2019).

[19] Hahn, S., Bode, M., Luttmann J. and Hoffmann, H.-D., "FULAS: high energy laser source for future lidar applications," Proc. SPIE 10562, 105620P (2017).

[20] Ehret, G., Bousquet, P., Pierangelo, C., Alpers, M., Millet, B., Abshire, J.B., Bovensmann, H., Burrows, J.P., Chevallier, F., Ciais, P., Crevoisier, C., Fix, A., Flamant, P., Frankenberg, C., Gibert, F., Heim, B., Heimann, M., Houweling, S., Hubberten, H.W., Jöckel, P., Law, K., Löw, A., Marshall, J., Agusti-Panareda, A., Payan, S., Prigent, C., Rairoux, P., Sachs, T., Scholze, M., Wirth, M., "MERLIN: A French-German Space Lidar Mission Dedicated to Atmospheric Methane," Remote Sens. 2017, 9, 1052 (2017).

[21] Livrozet, M., Gronloh, B., Faidel, H., Luttmann, J., Hoffmann, D., “Optical and Optomechanical Design of the MERLIN Laser Optical Bench,” ICSO2020, \#072 (2021).

[22] Löhring, J., Winzen, M., Faidel, H., Miesner, J., Plum, D., Klein, J., Fitzau, O., Giesberts, M., Brandenburg, W., Seidel, A., Schwanen, N., Riesters, D., Hengesbach, S. and Hoffmann, H.-D., "Key optical components for spaceborne lasers," Proc. SPIE 9730, 973000 (2016).

[23] Coney, A.T., and Damzen, M.J., "High-energy diode-pumped alexandrite amplifier development with applications in satellite-based lidar," J. Opt. Soc. Am. B 38, 209-219 (2021).

[24] Russbueldt, P., Hoffmann, D., Höfer, M., Löhring, J., Luttmann, J., Meissner, A., Weitenberg, J., Traub, M., Sartorius, T., Esser, D., Wester, R., Loosen, P., and Poprawe, R., "Innoslab Amplifiers," IEEE J. Sel. Topics Quantum Electron. 21, 447-463 (2015).

[25] Strotkamp, M., Elsen, F., Löhring, J., Traub, M., and Hoffmann, D., "Two stage Innoslab amplifier for energy scaling from 100 to $>500 \mathrm{~mJ}$ for future lidar applications," Appl. Opt. 56, 2886-2892 (2017).

[26] Kaifler, B., Büdenbender, C., Mahnke, P., Damm, M., Sauder, D., Kaifler, N. and Rapp, M., "Demonstration of an iron fluorescence lidar operating at $372 \mathrm{~nm}$ wavelength using a newly-developed Nd:YAG laser," Opt. Lett. 42, 2858-2861 (2017).

[27] Wester, R., "Physical optics methods for laser and nonlinear optics simulations," Advanced Optical Technologies, 2(3), 247-255 (2013). 